\title{
REFERENCES
}

1. Hung Ching Chow, On the summability factors of Fourier series, J. London Math. Soc. 16 (1941), 215-220.

2. Leonard McFadden, Absolute Nörlund summability, Duke Math. J. 9 (1942), 168-207.

3. T. Pati, The summability factors of infinite series, Duke Math. J. 21 (1954), 271-283.

4. B. N. Prasad and S. N. Bhatt, The summability factors of a Fourier series, Duke Math. J. 24 (1957), 103-117.

5. O. P. Varshney, On the absolute Harmonic summability of a series related to a Fourier series, Proc. Amer. Math. Soc. 10 (1959), 784-789.

Allahabad University, Allahabad, India

\section{ON THE COMPLETION OF TRACTABLE NORMED ALGEBRAS}

\author{
JOHN A. LINDBERG, JR.
}

A normed algebra $B$ (norm $\|\cdot\|$; we always assume that $B$ is commutative and possesses a multiplicative unit) is said to be tractable if the intersection of its $\|\cdot\|$-closed maximal ideals is trivial (cf. [1]). If $\bar{B}$ denotes the completion of $B$ with respect to $\|\cdot\|$, then we might ask if $\bar{B}$ can have a nontrivial radical. Examples have been given which show that this is indeed the case. In this note, we give a class of tractable normed algebras whose completions have nontrivial radicals and further show that it is possible to do this for certain algebras of functions which are complete in sup norm (but not all continuous functions on their carrier spaces). We make use of algebraic extensions of normed algebras and need the following results.

Lemma 1. If $A$ is a normed algebra (norm $\|\cdot\|$ ) and if $a \in A$, then $A[x] /\left(x^{2}-a\right)$ is a normed algebra under

$$
\left\|a_{0}+a_{1}+\left(x^{2}-a\right)\right\|_{1}=\left\|a_{0}\right\|+\left\|a_{1}\right\|\|a\| .
$$

LemMa 2. If $A$ is a tractable normed algebra and if $a \in A$, then $A[x] /\left(x^{2}-a\right)$ is a tractable normed algebra under $\|\cdot\|_{1}$ if and only if a is not a zero divisor in $A$.

LemMa 3. The $\|\cdot\|_{1}$-completion of $A[x] /\left(x^{2}-a\right), a \in A$, is (isomorphic to) $\bar{A}[x] /\left(x^{2}-a\right)$, where $\bar{A}$ denotes the $\|\cdot\|$-completion of $A$.

Lemmas 1 and 2 have been proved by Arens and Hoffman [1]. The

\footnotetext{
Received by the editors October 23, 1961 and, in revised form, February 23, 1962.
} 
proof of Lemma 3 is essentially the observation that $\left\{a_{0 n}+a_{1 n} x+\left(x^{2}-a\right)\right\}_{n=1}^{\infty}$ is a $\|\cdot\|_{1}$ Cauchy sequence if and only if the sequences $\left\{a_{0 n}\right\}_{n=1}^{\infty}$ and $\left\{a_{1 n}\right\}_{n=1}^{\infty}$ are $\|\cdot\|$-Cauchy sequences.

Suppose now that $A$ is a subalgebra of $C(\Omega)$, where $\Omega$ is a compact Hausdorff space. $A$ is supposed to contain the constant functions and separate the points of $\Omega$. If $J$ is a closed subset of $\Omega$, let $\|f\|_{J}$ denote $\max _{\omega \in J}|f(\omega)|$. If $O$ is the only function in $A$ vanishing on $J$, then $\|\cdot\|_{J}$ is clearly a norm on $A$ and $A$ is tractable under $\|\cdot\|_{J}$. If $a \in A$ and $J$ has the above property, then let $\|\cdot\|_{1, J}$ denote the norm on $A[x] /\left(x^{2}-a\right)$ which was specified in Lemma 1 . If $f \in A$, then let $N(f)=\{\omega \in \Omega: f(\omega)=0\}$.

TheORem. Let $A$ and $\Omega$ be as above. If $K$ is a closed subset of $\Omega$ such that $O$ is the only function in $A$ vanishing on $K$, if $f_{0} \in A$ and $N\left(f_{0}\right) \neq \varnothing$, and if there exists a sequence $\left\{f_{n}\right\} \subset A$ such that $f_{n} f_{0} \mid K \rightarrow 1$ (uniformly on $K)$, then $B=A[x] /\left(x^{2}-f_{0}\right)$ is tractable under $\|\cdot\|_{1, J}, J=K \cup N\left(f_{0}\right)$, and the $\|\cdot\|_{1, \mathrm{~J}}$-completion $\bar{B}$ of $B$ is not semi-simple; in fact, $\bar{B}=B_{0} \oplus R$ where $B_{0}$ is a closed subalgebra of $\bar{B}$ and $R$ is the radical of $\bar{B}$.

Proof. The algebra $B=A[x] /\left(x^{2}-f_{0}\right)$ is tractable under $\|\cdot\|_{1, J}$ since $f f_{0} \mid J=0, f \in A$, implies $f \mid K=0$ ( $f_{0}$ is never zero on $\left.K\right)$ and hence $f=0$.

Now, the completion $\bar{A}$ of $A$ is, of course, just a subalgebra of $C(J)$. Since $f_{n} f_{0}$ converges uniformly to one on $K$ and each $f_{n} f_{0}$ is identically zero on $N\left(f_{0}\right), f_{n} f_{0}$ must converge uniformly on $J$ to a function $e_{1} \in \bar{A}$, which must be an idempotent in $\bar{A}$. Let $e_{2}=e-e_{1}$, where $e$ is the function identically one on $\Omega$. Then $e_{2} f_{0}=0$. By Lemma $2, \bar{B}$ is not semi-simple (=tractable + complete).

We now show that $\bar{B}$ possesses a Wedderburn decomposition. It is easily shown that the radical $R$ of $\bar{B}$ is the set $\left\{a e_{2} x+\left(x^{2}-f_{0}\right): a \in \bar{A}\right\}$ (cf. [1]). Since

$a_{0}+a_{1} x+\left(x^{2}-f_{0}\right)=\left(a_{0}+a_{1} e_{1} x+\left(x^{2}-f_{0}\right)\right)+\left(a_{1} e_{2} x+\left(x^{2}-f_{0}\right)\right)$,

we have that $\bar{B}=\bar{A}\left[e_{1} x+\left(x^{2}-f_{0}\right)\right]+R$. Now, if $\left(a_{0}+a_{1} e_{1} x+\left(x^{2}-f_{0}\right)\right)$ $+\left(a e_{2} x+\left(x^{2}-f_{0}\right)\right)=0$, then $a_{0}=0$ and $a_{1} e_{1}+a e_{2}=0$. Since $e_{1} e_{2}=0$, $a_{1} e_{1}=0$ and $a e_{2}=0$ so that the above sum is a direct sum. To show that $B_{0}=\bar{A}\left[e_{1} x+\left(x^{2}-f_{0}\right)\right]$ is closed in $\bar{B}$, we consider a Cauchy sequence $\left\{a_{0 n}+a_{1 n} e_{1} x+\left(x^{2}-f_{0}\right)\right\}_{n=1}^{\infty}$ in $B_{0}$. Now, as a Cauchy sequence in $\bar{B}$, it must have a limit $a_{0}+a_{1} x+\left(x^{2}-f_{0}\right)$, where $a_{0}=\lim _{n \rightarrow \infty} a_{0 n}$ and $a_{1}=\lim _{n \rightarrow \infty} a_{1 n} e_{1}$. But this means that $a_{1} e_{1}=a_{1}$ so that $a_{0}+a_{1} x+\left(x^{2}-f_{0}\right)$ $\in B_{0}$. Hence $B_{0}$ is closed in $\bar{B}$. This completes the proof of the theorem.

Let $\Omega_{0}=\left\{(\omega, \lambda) \in \Omega \times C: \lambda^{2}=f_{0}(\omega)\right\} \quad$ and $\quad \Omega_{1}=\{(\omega, \lambda) \in J \times C$ : 
$\left.\lambda^{2}=f_{0}(\omega)\right\}$, where $C$ denotes the complex numbers. $\Omega_{0}$ and $\Omega_{1}$ are to be endowed with the relativized product topologies from $\Omega \times C$ and $J \times C$, respectively.

CoRollary. Maintain the hypotheses of the theorem. If in addition $A$ is closed is $C(\Omega)$ and if $f_{0}$ fails to vanish on $\partial_{A} \Omega$ (= Silov boundary of $A$; cf. [4]), then $B$ is isomorphic to a closed subalgebra of $C\left(\Omega_{0}\right)$ and $B_{0}$ is isomorphic to a closed subalgebra of $C\left(\Omega_{1}\right)$.

The first assertion follows from Theorem 1 of [2] and the second assertion follows from Theorem 2 of the same paper.

The conditions on $A$ are easily fulfilled. Let $A$ denote the algebra $\operatorname{Hol}(\Delta)$ of all functions continuous on $\Delta=\{z:|z| \leqq 1\}$ and analytic in the interior of $\Delta$. Let $K$ be the closed interval $\left[\frac{1}{2}, 1\right]$ and let $f_{0}(z)=z$, $z \in \Delta$. Since $A \mid K \cup N\left(f_{0}\right)$ is dense in $C\left(K \cup N\left(f_{0}\right)\right.$ ) (by the Weierstrass approximation theorem), the conditions of the corollary are easily shown to be satisfied. (In view of the theorem on polynomial approximation proved by Mergelyan [3], the conclusion of the corollary remains valid if $K$ is an infinite closed subset of the interior of $\Delta$ which is nowhere dense and does not separate the plane.)

Further consideration of the algebra $\operatorname{Hol}(\Delta)$ reveals that $\operatorname{Hol}(\Delta)$ and $B=(\operatorname{Hol}(\Delta))[x] /\left(x^{2}-f_{0}\right)$ are isomorphic in a natural way $\left(\left(g_{0}+g_{1} x+\left(x^{2}-f_{0}\right)\right) \rightarrow h_{0}+h_{1} f_{0}\right.$, where $h_{i}(z)=g_{i}\left(z^{2}\right)$, and $\left.g_{i} \in A, i=1,2\right)$ so that we have produced a norm on $\operatorname{Hol}(\Delta)$ with respect to which the completion is a Banach algebra with a nontrivial radical.

\section{REFERENCES}

1. R. Arens and K. Hoffman, Algebraic extensions of normed algebras, Proc. Amer. Math. Soc. 7 (1956), 203-210.

2. G. A. Heuer and J. A. Lindberg, Jr., Algebraic extensions of continuous function algebras, Proc. Amer. Math. Soc. 14 (1963), 337-342.

3. S. N. Mergelyan, On the representation of functions by series of polynomials, Dokl. Akad. Nauk SSSR 78 (1951), 405-408; Amer. Math. Soc. Transl. Series 1, no. 85. 1960.

4. C. E. Rickart, General theory of Banach algebras, Van Nostrand, New York,

YALE UNIVERSITY 